\title{
A probabilistic approach for links between rheumatic diseases and weather
}

\author{
Hiroshi Morimoto ${ }^{1 *}$ \\ ${ }^{1}$ The graduate school of informatics, Nagoya University, Japan
}

\begin{abstract}
Rheumatoid arthritis (RA) is an autoimmune disorder characterized by a painful swelling that can eventually result in bone erosion and joint deformity. Many works have studied the effect of weather on arthritis and rheumatism. However, some studies showed that the relationship was not clinically significant, and thus scientific evidence on the link remains sparse and non-conclusive. The aim of this paper is to clarify what sort of changes of weather factors lead to the deterioration of the symptom of RA by a probabilistic method in conjunction with cytokine network.

As a method, we chose a naive Bayes classifier rather than usual regression models. While a naive Bayes classifier is normally used for the problem of discrimination, we invented a new application of naive Bayes and applied it to calculate the tendency of meteorological factors towards high risk of deterioration of RA. Among meteorological factors, we selected barometric pressure and relative humidity.

We identified three patterns of tendencies of weather factors that led to deterioration of rheumatic diseases. These findings suggested mechanisms connecting weather and RA. The differences of three patterns were associated with the varied responses of cytokine network including interleukin IL-6 as a key cytokine. Our findings are expected to contribute to avoid the event of destructive deterioration of RA on the basis of daily observation of the weather.
\end{abstract}

\section{Introduction}

Rheumatoid arthritis (RA) is a chronic systemic disorder with unknown etiology, characterized by autoimmunity and progressive destruction of the small joints of the hands and feet. It is accompanied by pain, swelling and an increased risk of osteoporosis and coronary heart disease. Many medical writings have addressed the effect of weather on arthritis and rheumatism [1,2]. However some studies showed that the relationship was not clinically significant $[3,4]$ and thus the links between weather and rheumatism remains still unclear.

In this paper, we aimed to forecast the risk of worsening the symptom of rheumatoid arthritis by changes of two meteorological factors, barometric pressure and relative humidity, on the basis of probabilistic method such as naive Bayes classifier. In other word, we clarified what sort of changes of these two weather factors led to the deterioration of the symptom of RA.

We adopted, as a method, a naive Bayes classifier, while in most of epidemiological studies the regression models were used. A naive Bayes classifier is normally used for the problem of discrimination, but here we invented a new application of naive Bayes. A naive Bayes gives probabilistic distribution of risk, and we managed this distribution in order to calculate the tendency of meteorological factors towards high risk. Among meteorological factors, we selected barometric pressure and relative humidity, because these two factors were described to be associated with the rheumatoid arthritis in many studies $[1,2,5]$.

We identified tendencies of weather factors that led to deterioration of rheumatic diseases. These findings suggested a presumable mechanism that might have linked weather with RA. In the background mechanism of RA, there were chains of inflammatory responses in the pathogenesis of chronic destructive joint inflammation. We discussed the role of cytokine network as one of these inflammatory chains that lied in the mechanism of RA in connection with the effects of weather changes.

\section{Method}

The data of patients of rheumatoid arthritis were provided by the city of Nagoya. The data consisted of the daily data of the number of patients during two periods, from 2002 to 2005 and from 2009 to 2012. Nagoya is Japan's third-largest city and is located on the Pacific coast on central land of Japan. The climate of Nagoya is characterized by a standard subtropical climate with four characteristic annual seasons. The data included the number of patients of all ages, who were first transported by ambulance to a hospital and then diagnosed at the hospital with rheumatoid arthritis. We also used daily meteorological data, barometric pressure and relative humidity, extracted from Japan Meteorological Agency.

A naive Bayes classifier is a simple probabilistic classifier based on applying Bayes' theorem with independence assumptions of variables $[6,7,8]$. Let $\mathrm{C}$ be the random variable denoting the class of an instance, and $\mathrm{X}=\left(\mathrm{X}_{1}, \ldots, \mathrm{X}_{\mathrm{n}}\right)$ be an array of random variables denoting the observed attribute values. Let $\mathrm{c}$ be a particular class label and $\mathrm{x}=$ $\left(\mathrm{x}_{1}, \ldots, \mathrm{x}_{\mathrm{n}}\right)$ represent a particular observed attribute value. According to the independence assumption, attributes $\mathrm{X}_{1}, \ldots, \mathrm{X}_{\mathrm{n}}$ are all conditionally independent of one another, given $\mathrm{C}$. The value of this assumption is

Correspondence to: Hiroshi Morimoto, The graduate school of informatics, Nagoya University, Japan, E-mail: h.morimoto@i.nagoya-u.ac.jp or hiroshim@ info.human.nagoya-u.ac.jp

Key words: Bayes classifier, weather, rheumatoid arthritis, cytokine, interleukin

Received: April 22, 2017; Accepted: May 08, 2017; Published: May 13, 2017 
that it dramatically simplifies the representation of the conditional probability $\mathrm{P}(\mathrm{X} \mid \mathrm{C})$, and the problem of estimating it from the training data.

The probability of each class given the vector of observed values for the predictive attributes may be obtained using the Bayes' theorem:

$$
\mathrm{P}(\mathrm{C}=\mathrm{c} \mid \mathrm{X}=\mathrm{x})=\frac{P(C=c) P(X=x \mid C=c)}{P(X=x)}
$$

Because of the attributes conditional independence assumption, the following equation may be written:

$$
\left.\mathrm{P}(\mathrm{X}=\mathrm{x} \mid \mathrm{C}=\mathrm{c})=\prod_{i} P\left(X_{i}=x_{i}\right) \mid C=c\right)
$$

In the present work, $\mathrm{X}$ represented an array of meteorological factors. The variable $\mathrm{X}_{1}$ and $\mathrm{X}_{2}$ were set as daily data of barometric pressure and relative humidity. The variable $C$ expressed a risk of RA and was defined as a set of two classes of "high" risk and "low" risk for RA.

\section{Results}

A naive Bayes classifier is one of supervised classification algorithms to discriminate classes. Given observed values $X=\left(X_{1}, \ldots\right.$ ., $\mathrm{X}_{\mathrm{n}}$ ), a naive Bayes classifier guess the class $\mathrm{C}$ to which these values belong. Therefore this method was named "classifier". In the present work, we introduced a new application of these naive Bayes classifiers; by extracting "tendency" toward high risk of rheumatoid arthritis or "risk direction" from observed data.

We set $\mathrm{n}=2$ and selected daily data of barometric pressure as the variable $\mathrm{X}_{1}$ and humidity as $\mathrm{X}_{2}$. This defined observed variables $\mathrm{X}=\left(\mathrm{X}_{1}\right.$, $\mathrm{X}_{2}$ ). Daily data of barometric pressure and relative humidity in Nagoya city was extracted from Japan Meteorological Agency, during two terms 2002-2005 and 2009-2012.

\section{$\mathrm{X}=\left(\mathrm{X}_{1}, \mathrm{X}_{2}\right)=($ barometric pressure, relative humidity $)$}

We modified the data of patients of RA and designed the variable $\mathrm{C}$ denoting two classes of "high" risk and "low" risk for RA as follows. The daily data of the number of patients of RA were provided by the city of Nagoya, consisting of terms during two periods, from 2002 to 2005 and from 2009 to 2012 . We defined high risk class $\mathrm{c} 1$ if the number of patients was greater or equal to 1 . Similarly, we defined low risk class $c_{2}$ if the number of patients was equal to 0 .

\section{$\mathrm{C}=\left\{\mathrm{c}_{1}, \mathrm{c}_{2}\right\}=\{$ high risk, low risk $\}$}

Figure 1 described the framework of a naive Bayes classifier. A naive Bayes classifier was also considered as a simple case of Bayesian networks.

From these definitions, the observed data of barometric pressure and humidity $\mathrm{x}=\left(\mathrm{x}_{1}, \mathrm{x}_{2}\right)$ at some day could be considered as a point in the plane $\mathrm{X}=\left(\mathrm{X}_{1}, \mathrm{X}_{2}\right)$. The class $\mathrm{C}$ (high risk or low risk) at the same day was regarded as a kind of value over the point $\mathrm{x}=\left(\mathrm{x}_{1}, \mathrm{x}_{2}\right)$. These values over points were called training data or teacher signal. We selected arbitrary ten values from the interval $\left[\min \left(\mathrm{X}_{1}\right), \max \left(\mathrm{X}_{1}\right)\right]$, and other ten values from $\left[\min \left(\mathrm{X}_{2}\right), \max \left(\mathrm{X}_{2}\right)\right]$. We then considered $10 \times 10$ lattices $\mathrm{L}$ in the plane $\mathrm{X}=\left(\mathrm{X}_{1}, \mathrm{X}_{2}\right)$ formed by crossing these ten values on each variables $\mathrm{X}_{1}$ and $\mathrm{X}_{2}$. A naive Bayes classifier is a method that provides "extension" of training data to the lattices $\mathrm{L}$.

Given a point $\mathrm{x}$ in lattices $\mathrm{L}$, i.e., given a pair of values $\left(\mathrm{x}_{1}, \mathrm{x}_{2}\right)$ of barometric pressure and humidity, a naive Bayes classifier could calculate the probability that the risk for RA became "high" at that point. Collecting all of these values over the lattices $L$, we could get the distribution of probabilities (probability distribution) over L. Since

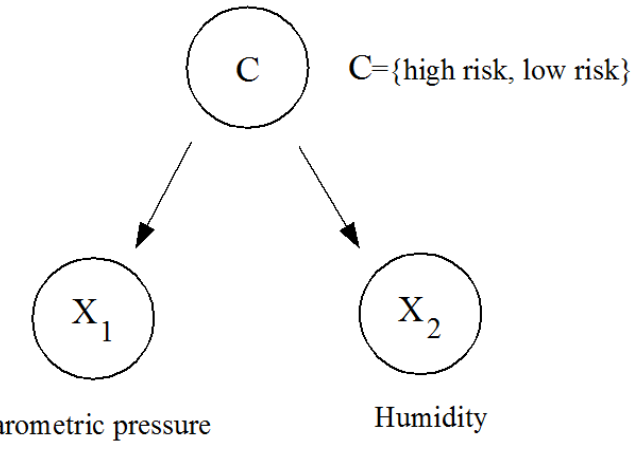

Figure 1. A description of a naive Bayes classifier. Given the values of barometric pressure and relative humidity, the probability, $\mathrm{P}(C \mid X)$, that the event of destructive phase of rheumatic arthritis occurs can be calculated by Bayes' theorem from $\mathrm{P}(X \mid C)$.

this distribution could be considered as a function over the plane, this enabled us to get gradient vectors (i.e., a sort of tilts) of this function. These vectors expressed the directions along which the risk for RA increased mostly. Thus these vectors could be called "risk direction" or "tendency towards high risk".

These vectors of risk directions are shown in Figure 2. In these figures, the horizontal lines implied barometric pressure, and the vertical line humidity. The curves were contour lines of probability distribution. The vectors meant the "risk direction", i.e., the direction along which the risk increased. Observing these vectors of directions, we could capture an overall trend towards the high risk when barometric pressure and humidity changed.

We explored all the risk directions for January, March, July, October and November during two terms, 2002-2005 and 20092012, respectively. These months were selected because in Nagoya the patients of RA who were transported to hospitals by emergency vehicles scored high levels on these months. From the calculations of "risk directions", we found the following three main patterns (a), (b) and (c) of risk directions:

(a) If the barometric pressure decreases, the risk increases,

(b) If the humidity increases, the risk increases and

(c) If the barometric pressure decreases and the humidity increases at the same time, then the risk of RA increases

In Figure 2, (a) was for November in 2010, (b) for March in 2003, and (c) for July in 2010.

In viewing these results, we could note that the change of barometric pressure and humidity were closely related to the change of risk towards deterioration of RA. The differences of these patterns suggested us to address the background mechanism that linked the weather and RA. This mechanism was discussed in discussion section with connection to cytokine network of inflammatory response including various interleukins.

\section{Discussion}

Given the values of barometric pressure and relative humidity, we calculated the probability of the risk towards deterioration of rheumatic diseases, applying naive Bayes classifier. Over the plane of two variables, barometric pressure and relative humidity, we calculated the distribution of this sort of probabilities. 
(a)risks in 2010, month $=11$, delay $=3$

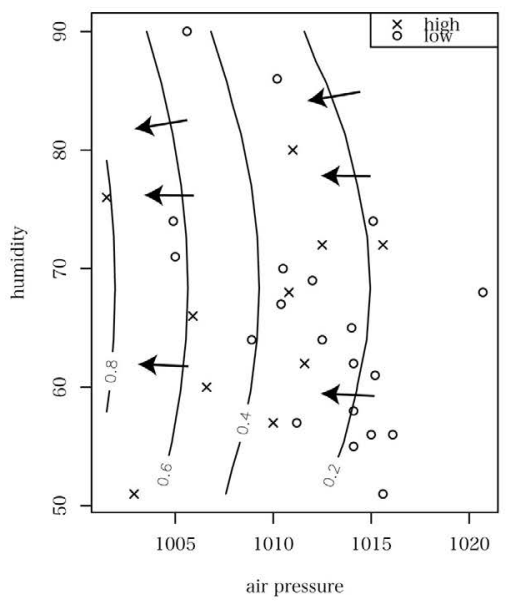

(b)risks in 2003 , month $=3$, delay $=3$

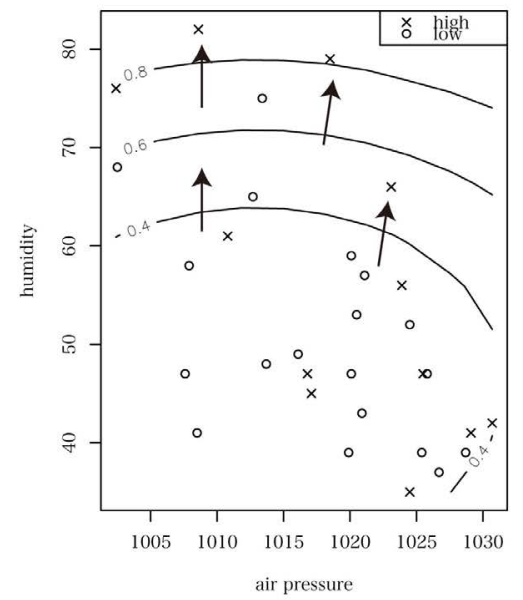

(c)risks in 2010 , month $=7$, delay $=3$

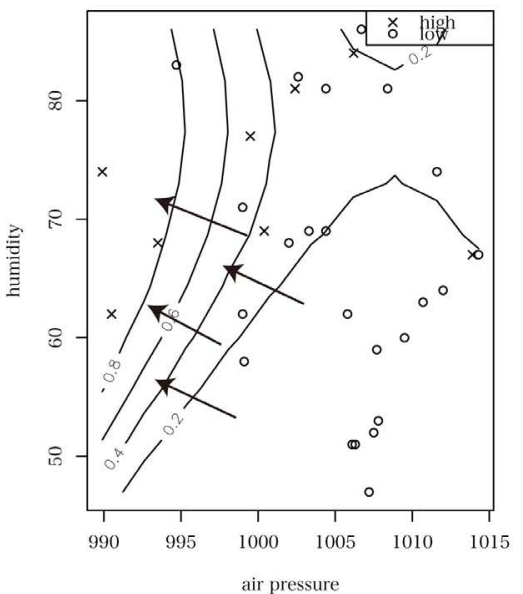

Figure 2. The vectors of "risk directions" are described. The horizontal lines express barometric pressure, and the vertical lines relative humidity. The curves were contour lines of the function of probability distribution. The vectors mean the direction along which the risk increases. (a) only the decrease of barometric pressure causes risk, (b) only the decrease of relative humidity causes risk and (c) both barometric pressure and relative humidity together causes risk.

We could extract from this distribution the vectors that expressed the directions along which the risk for RA increased. Thus, these vectors were called "risk direction".

We explored these risk directions for two terms, 2002-2005 and 2009-2012, respectively. We identified three main patterns of risk directions, where the decrease of barometric pressures and the increase of relative humidity were associated with the risk of rheumatic diseases.

Figure 2 (a) shows that the decrease of barometric pressure is related to the deterioration of RA. This finding supports the result of the work [5] that reported who RA disease activity revealed suggestive negative associations with barometric pressure. Furthermore, they asserted that barometric pressure was the only weather factor associated with RA and that the association was independent from humidity and temperature. This corresponds exactly with the picture of Figure 2(a).

The result is also interesting from a viewpoint of molecular levels or gene expression levels. Since rheumatic disease is one of autoimmune diseases, it is related to immune reaction, inflammatory responses and cytokine network. Therefore, relation between weather and cytokines becomes an important problem in order to identify mechanisms behind rheumatic diseases. Our finding of the pattern (a) is closely related to cytokines including tumour necrosis factor (TNF-alpha) and interleukin 6 (IL-6). If the barometric pressure decreases, the hydrostatic pressure (HP) between cells increases, causing edema or swelling. The increase of HP induced mRNA expressions of IL-6 and TNF- alpha [9]. Our pattern (a) is also related to interleukin 8 (IL8). It was reported that mechanical stress induced expression of IL-8 and IL-6 in human periodontal ligament cells [10]. Mast cells may be influenced by barometric pressure and be an important source of TNFalpha and IL-6 [11].

Figure 2 (b) shows that the increase of relative humidity raises the risk of RA. This finding is related to the work [12]. At hot circumstance, an increase in relative humidity resulted in the hypomethylation of intercellular adhesion molecule-1 (ICAM-1) and was suggested to increase the production of the ICAM-1 protein. ICAM-1 encodes a cell surface glycoprotein which is typically expressed on endothelial cells and cells of the immune system. ICAM-1 is overexpressed during inflammatory responses. Relative humidity was also associated with interferon gamma (IFN-gamma). IFN-gamma is known to intensify inflammation.

Figure 2 (c) shows that the decrease of barometric pressure and the increase of humidity are associated with the risk of RA. This finding supports the result of the research [1]. Hollander conducted an experiment by putting humans in a chamber, called "climate chamber" where meteorological parameters were regulated. Patients with obvious physical signs of arthritis were observed in the Climate Chamber in pairs for periods of at least two weeks each. They found that barometric and humidity variations together were the only climatic parameters responsible for increased arthritic activity. Furthermore, the research work showed that rain influenced fibromyalgia [2].

We observed that our findings of patterns (a), (b) and (c) were closely connected to cytokine, especially interleukin. Autoimmune diseases are regulated by a variety of cytokine networks. Cytokines are cell signalling molecules that aid cell to cell communication in immune responses and stimulate the movement of cells towards sites of inflammation, infection and trauma. Cytokines exist in peptide, protein and glycoprotein (proteins with a sugar attached) forms. Interleukins (IL) are a subset of cytokines, and are related to many diseases, particularly in immune system like autoimmune diseases.

IL-6 is a cytokine involved in a wide variety of biological functions and is a key mediator in diseases like rheumatic diseases. IL- 6 knockout mice have been shown to be protected from developing joint symptoms in an arthritis model in vivo [13].

We summarized cytokine network involved in rheumatic diseases in Figure 3. Mast cell results in rapid release of TNF-alpha, inducing IL-6 in infiltrating mononuclear cells 11. TNF- alpha of mast cell origin may be a crucial factor in upregulating IL-6 [14]. IL-1 and TNF-alpha are pro-inflammatory mediators at several joint cells including synovial fibroblast, macrophages, and chondrocytes. They induce pain, swelling and progressive destruction of the small joints of the hands and feet, accompanied by loss of function and promote cartilage destruction and bone erosion in experimental arthritis.

We can notice a cascade of positive feedback in Figure 3 including IL-17 and IL-6. IL-17 is known to stimulate the expression of IL-6, IL- 


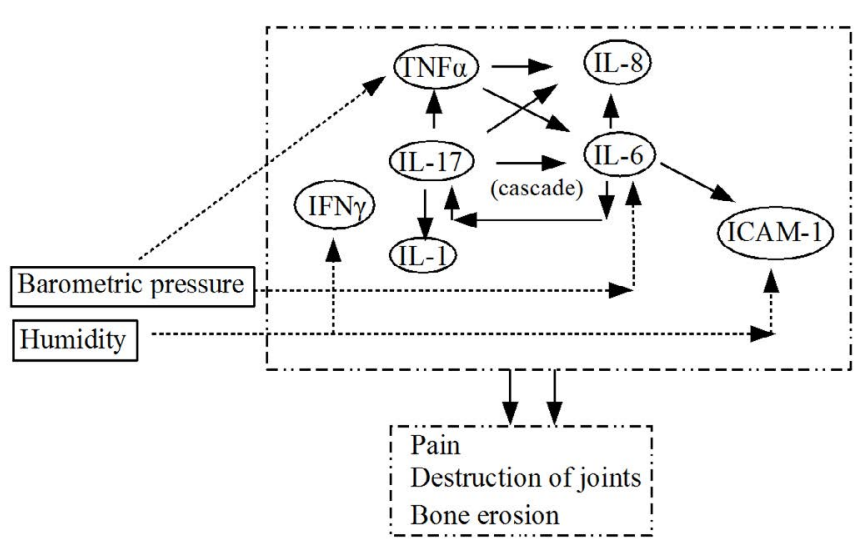

Figure 3. A description of a network of cytokines, consisting of TNF-alpha, IFN-gamma, ICAM-1 and interleukins IL-1, IL-6, IL-8 and IL-17, together with connections to weather factors and destructive state of RA. One should notice the existence of cascade loop including IL-6 and IL-17.

8, IL-1 and TNF-alpha in fibroblastic, endothelial, and epithelial cells [15]. IL-17 induces the production of pro-inflammatory mediators, such as IL-1 and TNF-alpha from several joint cells including synovial fibroblast, macrophages, and chondrocytes. IL-17 may play a role in fine-tuning the inflammatory response. There is an IL-17-triggered positive-feedback loop of IL-6 signaling [16]. If this cascade including IL-17 and IL-6 is driven, then this may result in pain, swelling and progressive destruction of the small joints of the hands and feet, accompanied by loss of function. They described the role of this novel cytokine family in the pathogenesis of chronic destructive joint inflammation.

Summarizing these findings, we can suggest the following mechanism that connects weather and rheumatic diseases. If the change of weather occurs, particularly in barometric pressure and relative humidity, then it stimulates the expression of IL- 6 and drives a cascade of positive feedback loop with IL-17. This may result in destructive arthritis finally.

\section{Conclusion}

Applying naive Bayes classifier to the data of meteorological factors and the data of patients of rheumatoid arthritis (RA), we proposed a new method to capture the overall trend towards the deterioration of RA by introducing the notion of "risk directions". Three typical patterns of tendency to lead to the destruction of RA were identified (types (a), (b) and (c) in Figure 2) as patterns of "risk directions". These patterns included the influence of only one or both of the decrease of barometric pressure and the increase of relative humidity. The sole pattern (a) or (c) was already found by other works. However, our method can capture these types together within a scope of the notion of "risk directions". These findings suggested the importance of the role of cytokine network that connects weather and RA, especially the importance of the cascade loop including interleukins IL-6 and IL-17 (Figure 3). IL-6 is a key cytokine in inflammatory responses and its gene expression may be influenced by barometric pressure. Our findings will play an important role in avoiding pathogenesis of chronic destructive joint inflammation by daily observation of weather.

\section{References}

1. Hollander JP, Yeostros SY (1963) The Effect of Simultaneous Variations of Humidity and Barometric Pressure on Arthritis. AIBS Bulletin 13: 24-28.

2. Guedj D, Weinberger A (1990) Effect of weather conditions on rheumatic patients. Ann Rheum Dis 49: 158-159. [Crossref]

3. Gorina AA, Smytha JM, Weisbergb JN, Affleckc G, Tennenc H, et al. (1999) Rheumatoid arthritis patients show weather sensitivity in daily life, but the relationship is not clinically significant. Pain 81: 173-177. [Crossref]

4. Fors EA, Sexton H (2002) Weather and the pain in fibromyalgia: are they related? Ann Rheum Dis 61: 247-250. [Crossref]

5. Terao C, Hashimoto M, Furu M, Nakabo S, Ohmura K, et al. (2014) Inverse Association between Air Pressure and Rheumatoid Arthritis Synovitis. PLOS ONE 9: 1-7. [Crossref]

6. Albert, Chi S (1993) Bayesian Analysis of Binary and Polychotomous Response Data Journal of the American Statistical Association. 88: 669-679.

7. Soria D, Garibaldi JM, Ambrogi F, Biganzoli EM, Ellis IO (2011) A 'non-parametric' version of the naive Bayes classifier. Knowledge-Based Systems 24: 775-784.

8. Morimoto H (2017) Suggestions for weather index to discriminate risks of cerebral and myocardial infarction. Journal of General and Emergency Medicine 2: 1-8.

9. Takahashi K, Kubo T, Arai Y, Kitajima I, Takigawa M, et al. (1988) Hydrostatic pressure induces expression of interleukin 6 and tumour necrosis factor alpha mRNAs in a chondrocyte-like cell line. Ann Rheum Dis 57: 231-236.

10. Yamamoto T, Kita M, Kimura I, Oseko F, Terauchi R, et al. (2006) Mechanical stres induces expression of cytokines in human periodontal ligament cells Oral Diseases 12: 171-175. [Crossref]

11. Frangogiannisa NG, Smithb CW, Entman ML (2002) The inflammatory response in myocardial infarction. Cardiovascular Research 53: 31-47. [Crossref]

12. Bind MA, Zanobetti A, Gasparrini A (2014) Effects of Temperature and Relative Humidity on DNA Methylation. Epidemiology 25: 561-569. [Crossref]

13. Emery P, Keystone E, P Tony H, Cantagrel A, van Vollenhoven R, et al. (2009) IL-6 receptor inhibition with tocilizumab improves treatment outcomes in patients with rheumatoid arthritis refractory to anti-tumour necrosis factor biologicals: results from a 24-week multicentre randomised placebo-controlled trial. Annals of the Rheumatic Diseases 67: 1-10.

14. Pang G, Couch L, Batey R, Clancy R, Cripps A (1994) GM-CSF, IL-1 alpha,IL-1 beta,IL-6,IL-8,IL-10,ICAM-1 and VCAM-1 gene expression and cytokine production in human duodenal fibroblasts stimulated with lipopolysaccharide, IL-1 alpha and TNFalpha. Clin Exp Immunol 96: 437-443. [Crossref]

15. Lubberts E (2008) IL-17/Th17 targeting: On the road to prevent chronic destructive arthritis. Cytokine 41: 84-91. [Crossref]

16. Ogura H, Murakami M, Okuyama Y, Tsuruoka M, Kitabayashi C, et al. (2008) Interleukin-17 Promotes Autoimmunity by Triggering a Positive-Feedback Loop via Interleukin-6 Induction. Immunity 29: 628-636. [Crossref]

Copyright: (C2017 Morimoto H. This is an open-access article distributed under the terms of the Creative Commons Attribution License, which permits unrestricted use, distribution, and reproduction in any medium, provided the original author and source are credited. 